\title{
Advantages of Low-kV TEM in the Study of Beam Sensitive Materials
}

Toshie Yaguchi ${ }^{1}$, Jim Kilcrease $^{2}$, Keisuke Igarashi ${ }^{1}$, Akiko Wakui ${ }^{1}$ and Keiji Tamura ${ }^{1}$

${ }^{1}$ Hitachi High-Tech Corporation, Hitachinaka-shi, Ibaraki, Japan, ${ }^{2}$ Hitachi High-Tech America Inc, Clarksburg, Maryland, United States

Low $\mathrm{kV}$ transmission electron microscopy (TEM) has been widely utilized in the biological and soft material fields due to many advantageous features including high contrast, high versatility and lowdamage imaging. Such features are also beneficial in the characterization of sensitive composite materials used in many today's cutting-edge battery technologies. Therefore, to better address the expanding classes of electron beam sensitive materials, new features have been added to the HT7800 $120 \mathrm{kV}$ TEM series platform. One such implementation is outfitting of an optimized field limiting aperture in order to obtain electron diffraction patterns under the same low-dose conditions that are utilized for TEM observation of samples prone to beam damage.

The nano-area aperture allows for collection of low-dose diffraction patterns from a sample region that is approximately $18 \mathrm{~nm}$ in diameter [1]. In conjunction with this feature, the utilization of hollow cone dark filed (HCDF) imaging with an air-protection heating holder can now be routinely applied for in-situ studies such as the crystallization of glass solid electrolytes for all-solid-state lithium batteries [2].

In this study, we characterize the method for analyzing beam sensitive materials using low acceleration voltage TEM. To accomplish this, we utilized a high-sensitivity CMOS camera to enable accurate viewing / navigation of specimens even under very low beam intensities which would otherwise present a great challenge for a traditional phosphorous viewing chamber. A comparison has been carried out for visualization of Pt (Platinum) - GC (Graphitized Carbon) catalyst with the electron current density of 1.03 $\mathrm{pA} / \mathrm{cm}^{2}$, between a image without any adjustments (analogous to traditional phosphorous chamber viewing) and one with digital gain adjustments for much easier visualization, especially under normal room light conditions. If current is reduced even further, visualization is still possible by increasing the screen camera gain, whereby under these conditions using a traditional phosphor chamber and 'naked eye navigation' it may not be possible.

Operation at low accelerating voltages provides higher scattering contrast as well as less knock-on damage. Fig. 1 shows TEM images of single walled carbon nanotubes from $20 \mathrm{kV}$ to $120 \mathrm{kV}$. Fine detail of the nanotubes is clearly distinguished with excellent contrast at lower accelerating voltage.

Minimization of beam dose is also necessary for the observation of organic, polymeric, and ionic materials. Fig. 2 demonstrates TEM images of ionomer coated Pt/CB (Carbon Black) electrocatalyst from a fuel cell, observed at $120 \mathrm{kV}$. A TEM image of ionomer with high contrast and low damage is shown in (a) whereby the dashed line shows the outline of the ionomer coated Pt/CB electrocatalyst. Due to higher beam dose, the ionomer area shrank after dosing as shown in (b). It is revealed that beam dose must be carefully monitored and damage mitigated imaging of materials using low $\mathrm{kV}$ is very advantageous. 

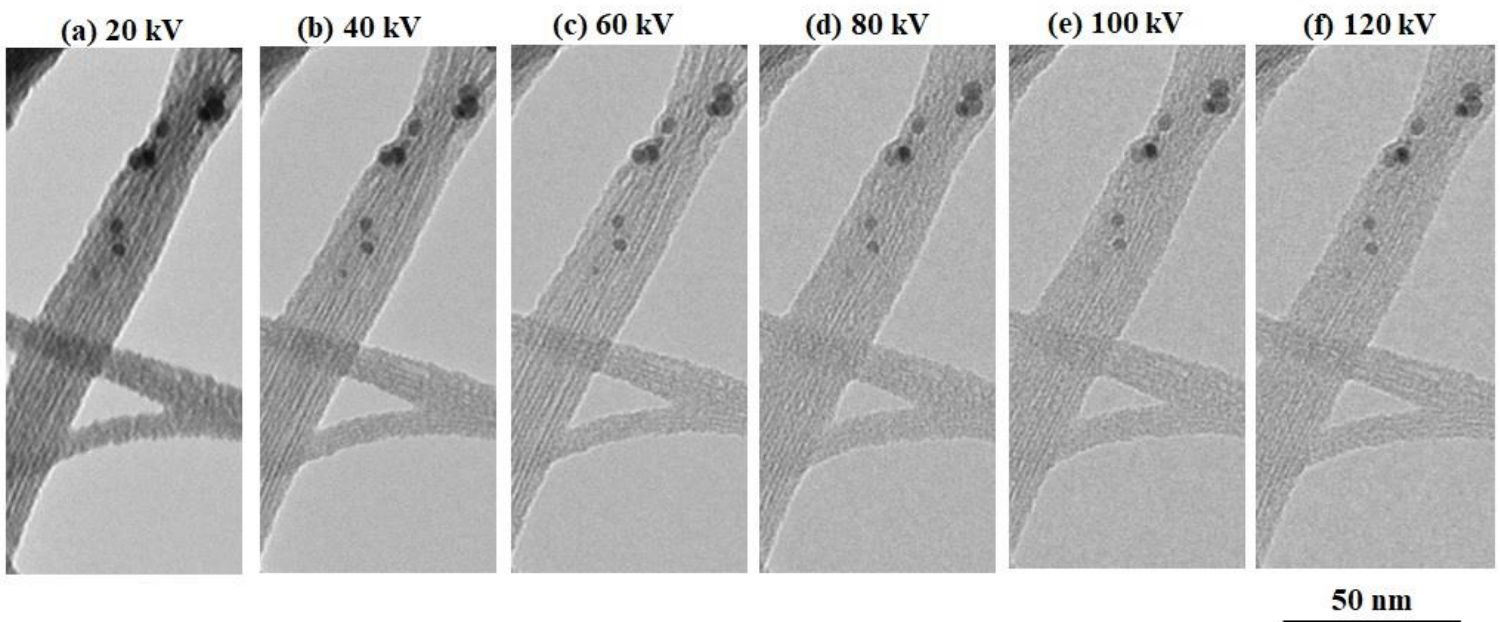

Figure 1. TEM images of single walled carbon nanotubes at the ranges from $20 \mathrm{kV}$ to $120 \mathrm{kV}$.
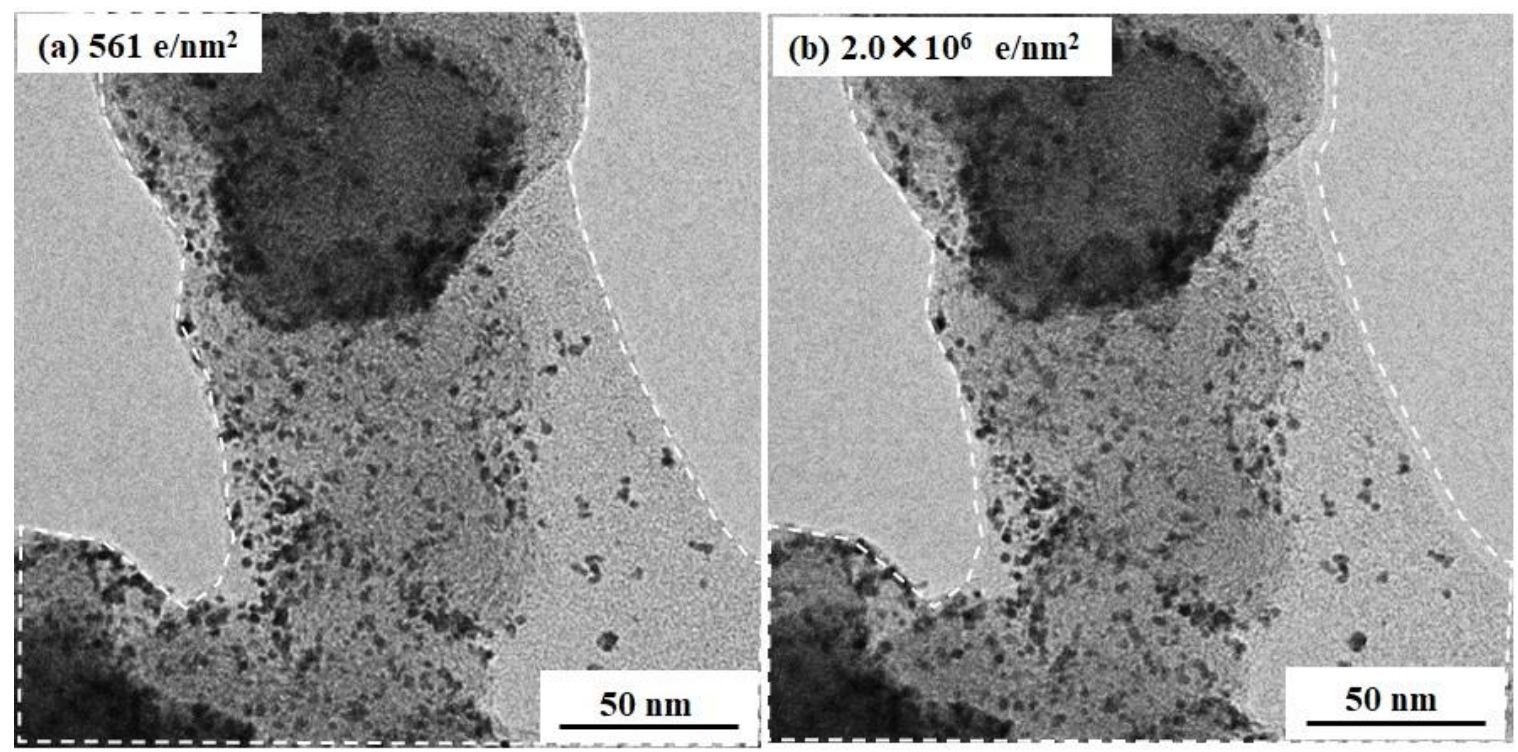

Figure 2. TEM images of ionomer coated Pt/CB (Carbon Black) electrocatalyst from a fuel cell, observed at $120 \mathrm{kV}$. A TEM image of ionomer with high contrast and low damage is shown in (a). The dashed line shows the outline of the ionomer coated Pt/CB electrocatalyst in (a). Due to higher beam dose, the ionomer area shrank after dosing as shown in (b).

\section{References}

[1] K.Tamura, et al, Microsc. Microanal. 24 (Suppl 1), 2018, 1156-1157.

[2] Igarashi et al., Proceeding of the 60th Battery Symposium, Japan. p.215. (2019). 